\title{
ORIGIN OF BINARIES FROM A CONSIDERATION OF ANGULAR MOMENTUM
}

\author{
Su-SHu Huang \\ (Dept. of Astronomy, Northwestern University, Evanston, Ill., U.S.A.)
}

\begin{abstract}
According to various studies, more than one half of stars exist as members of binary and multiple systems. Therefore any theory for star formation must account for this fact. In other words, the problem of the origin of binaries has a far broader implication than is implied as a part of binary-star study. For this reason one cannot overexaggerate its importance.
\end{abstract}

\section{Observed Results}

Statistical studies of binary stars have been proven to be useful for understanding stellar evolution (Hynek, 1967). In order to discuss their origin we should similarly examine the statistical results. Two facts which will be briefly reviewed here appear to be crucial:

\section{A. ORIENTATION OF ANGULAR MOMENTUM VECTORS}

The early investigations on this problem were confined to visual binaries, because they are the only kind of binaries whose orientation in space may be ascertained by observation. Even so, the actual determination of the orientation of a visual binary is not an easy task, and orbital planes of only a limited number of visual binaries have been determined. Chang (1929), who collected 16 visual binaries with their inclinations definitely established, found a random orientation in space of orbital planes. Later Finsen (1933) undertook a similar study of 28 visual binaries and obtained the same conclusion. Few additional determinations of the orbital orientation of visual binaries have been performed in recent years. Perhaps their conclusion may still be regarded as representing the final result along this line of study.

While both Chang and Finsen used a small number of systems with their orientations definitely ascertained, later investigators were inclined to rely on a large number of binaries for which the sign of the inclination, $i$, is not determined. A review of their methods and results has been given by Huang (1968). Most investigators obtained the same conclusion of random orientations, although there were some dissensions.

While it is difficult to find the sign of $i$ for visual orbits, it is not even possible to determine the magnitude of $i$ for spectroscopic binaries. It may be argued that the velocity curve of a spectroscopic binary depends upon the orbital inclination. A factor of $\sin i$ enters into the amplitude of the curve. This fact may aid us to detect

Perek (ed.), Highlights of Astronomy, 443-450. (.) I.A.U. 
any preferential orientation of these binaries from the statistics of the velocity-curve amplitudes of stars in different regions of the sky. Actually the problem is complicated by the systematic variation of stellar mass from the galactic equator to both poles, because the velocity-curve amplitude depends also upon the stellar mass (Shajn, 1926; Struve, 1950; Abt, 1966).

Although it is difficult to infer orbital orientations for spectroscopic binaries, a different situation obtains when they show the sign of eclipse. For then the inclination will be close to $\pi / 2$. Because of our own special location in the Galaxy, the inclination of the orbital plane of an eclipsing binary at galactic latitude, $b$, with the galactic plane can assume any value from $b$ to $\pi / 2$. Therefore, orbital planes of eclipsing binaries found in high galactic latitude necessarily make large angles with the galactic plane, while those found in low galactic latitude may make either large or small angles.

Because of this fact one can examine whether orbital planes of eclipsing binaries show any preferential orientation parallel to the galactic plane. Based on the data collected by Koch et al. (1963) which cover known eclipsing binaries over the entire sky down to the 13th magnitude at minimum light, a statistical study (Huang and Wade, 1966) shows that there is no such preferential orientation.

\section{B. DISTRIBUTION OF BINARY SEPARATIONS}

The histogram in the figure obtained by Kuiper (1935) represents the frequency distribution of binaries in a volume of space around the Sun as against $\log a$, where $a$ denotes the semi-major axis of the relative orbits expressed in AU. It combines the statistical data of eclipsing, spectroscopic and visual binaries as well as commonproper-motion pairs. However, it excludes binaries whose combined brightnesses are below absolute magnitude 6.45 , and the differences in brightness of those two components are less than 4 mag. $K$ and $M$ giants are excluded from the statistics because they do not follow the usual mass-luminosity relation which has been used in order to derive $a$. How much Kuiper's histogram will be modified by Shapley's (1948) conclusion about the high abundance of W UMa systems is not clear at present.

Because of the difficulty involved in collecting statistical data, the histogram is not expected to represent an accurate picture of the distribution of binary separations. However it does give a general trend and serves as a test for any theory or theories that concern formation of binaries.

\section{Theories}

The random orientation of angular momenta of binaries gives us a clue as regards their origin. They must be the result of random processes. Consider an interstellar medium in which turbulence prevails. One can imagine a velocity field composed of random motions of eddies of different sizes. When a stellar condensation is formed in 
it, what is the net effect of all these eddy motions on the final stellar angular momentum? It is obvious that angular momenta due to motions of small eddies will cancel off. But the net angular momentum due to large eddies will in general be different from zero. Hence an amount of angular momentum is acquired by simply collecting matter in a medium in turbulent motion. Being a random process such a mode of acquisition inevitably leads to a Maxwellian distribution of stellar angular momenta (Kuiper, 1955; McCrea, 1959). If we write $\Omega=x \Omega_{\mathrm{p}}$ where $\Omega_{\mathrm{p}}$ denotes the most probable value of stellar angular momentum, the distribution of $x$ may be written as

$$
\phi(x) \mathrm{d} x=\frac{4}{\sqrt{\pi}} x^{2} e^{-x^{2}} \mathrm{~d} x
$$

The orbital angular momentum of a binary is given by

$$
\Omega=M_{1} M_{2}\left[\frac{G a\left(1-e^{2}\right)}{M_{1}+M_{2}}\right]^{1 / 2},
$$

where $M_{1}$ and $M_{2}$ stand respectively for the masses of two components, $e$ the orbital eccentricity and $G$ the gravitational constant. By neglecting the variations of $M_{1}, M_{2}$ and $e$, from one binary to another, Kuiper (1955) assumes $x=\alpha_{1} a^{1 / 2}$, where $\alpha_{1}$ is a constant. If we further neglect the spin-angular momentum, $\Omega$ as given by Equation (2) will be distributed according to Equation (1). From this condition we may write

$$
F(\log a) \mathrm{d} \log a=\phi(x) \mathrm{d} x,
$$

where $F(\log a)$ represents the distribution of $\log a$ as consistent with the histogram in the figure and is given by (Kuiper, 1955)

$$
F(\log a)=\frac{x \phi(x)}{2 \log e} .
$$

The value of $\alpha_{1}$ can be determined by fitting the calculated maximum of $F(\log a)$ with the observed maximum shown in the histogram. In this way $F(\log a)$ can be computed according to Equation (4) and is shown as the broken curve in Figure 1.

Kuiper's statistical calculation is incomplete because he has neglected the variation of $M_{1}, M_{2}$ and $e$. Also there is the question about the distribution function of $\Omega$. We shall first discuss the latter point. Stars are believed to be formed in groups (Roberts, 1957). Each emerges from an interstellar medium which has its own characteristic turbulent-velocity field. Therefore the reasoning that the angular moments are distributed according to the Maxwellian law is correct only for binaries in a single group. For different groups, the values of $\Omega_{\mathrm{p}}$ will be different because the turbulent velocity fields of the pre-stellar media are different.

Let us assume that $\Omega_{\mathrm{p}}$ itself follows the Maxwellian distribution with the most probable value $\Omega_{0}$. Perhaps such an assumption overstates the case. However, we will 
examine its effect as a preliminary study. If we write $\Omega=x \Omega_{0}$, it can be easily shown that the distribution function now becomes

$$
\phi(x) \mathrm{d} x=\frac{16}{\pi} x^{2} \mathrm{~d} x \int_{0}^{\infty} e^{-(x / y)^{2}-y^{2}} \frac{\mathrm{d} y}{y} .
$$

When $\phi(x)$ as given by this equation is substituted into Equation (4), we obtain the broken curve interspersed with dots, as is shown in Figure 1. Thus the discrepancy between the observed and calculated distribution is much reduced by assuming star formation in groups.

The variation of $a$ due to $e$ may be regarded as small. This is especially true for

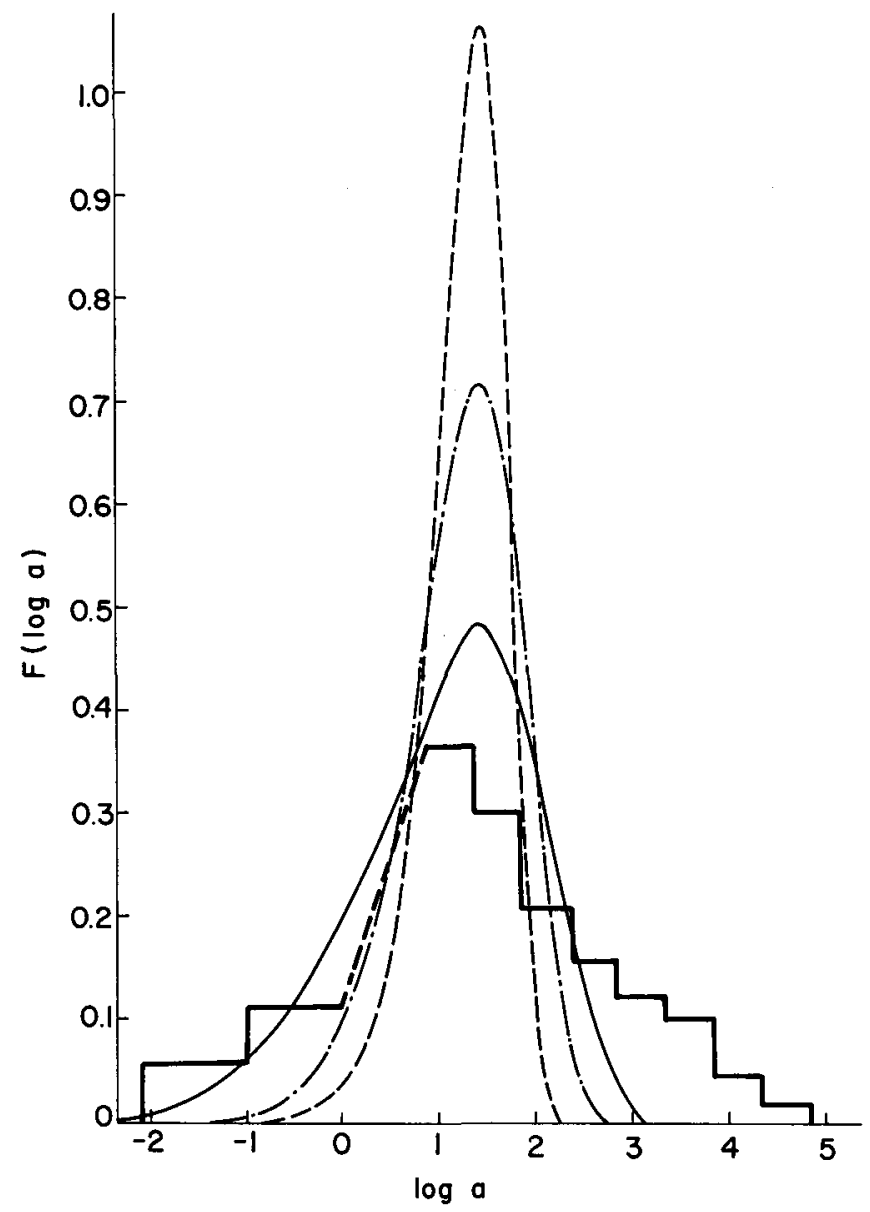

FIG. 1. Distribution of binary separations according to observational data (histogram) as well as statistical theories (curves) discussed in the paper. 
close binaries. Therefore we may neglect the effect of $e$ by setting it equal to zero everywhere. At the same time, instead of treating $M_{1}$ and $M_{2}$ directly we may use $y$ and $z$, defined by

$$
y=\frac{M_{2}}{M_{1}+M_{2}} \quad \text { and } \quad z=\frac{M_{1}+M_{2}}{M_{\odot}}
$$

as two independent variables. It may be argued that the variation of $y$ in observable binaries is limited to perhaps between 0.2 and 0.5 , while the range of variation of $z$ is much wider, say from 0.4 to 6 . Therefore the main contribution to the variation of $t$ is due to that of $x$ and $z$. We may propose to derive the distribution of $\log a$ from the distribution of $x$, given by Equation (5) and the distribution of $z$ which is assumed to follow a power law, namely

$$
\Psi(z) \mathrm{d} z=\frac{B \mathrm{~d} z}{z^{m}}, \quad z_{0} \leqslant z \leqslant z_{1},
$$

where $B$ is a normalizing factor and can be easily obtained. As an approximation we may take $m=2 \cdot 35$, which was obtained for single stars (Salpeter, 1955).

Instead of looking for the distribution of $a$, we may equivalently examine the distribution of $t$, defined by

$$
t=\alpha_{2} a \text { with } \quad \alpha_{2}=y^{2}(1-y)^{2} / \lambda^{2},
$$

where $\lambda$ represents a dimensionless parameter given by

$$
\lambda=\frac{\Omega_{0}}{\left(G a_{1} M_{\odot}^{3}\right)^{1 / 2}},
$$

if $a$ in Equation (8) is understood to be expressed in units of $a$, which may be taken as 1 AU. Hence $t$ differs from $a$ only by a constant factor since $y$ is not assumed to vary. The distribution function $F(t)$ of $t$ can now be expressed as a double integral (e.g., Chandrasekhar and Münch, 1950), namely

$$
f(t) \mathrm{d} t=\iint_{4} \phi(x) \mathrm{d} x \Psi(z) \mathrm{d} z,
$$

where the integration domain, $\Delta$, is defined by

$$
t \leqslant \frac{x^{2}}{z^{3}}<t+\mathrm{d} t
$$

in the $x-z$ plane.

Substitution of Equations (5) and (7) into Equation (10) and simple reduction of the resulting equation yield the normalized distribution of $t$ :

$$
f(t) \mathrm{d} t=\frac{8 B}{\pi} t^{1 / 2} \mathrm{~d} t \int_{z_{0}}^{z_{1}} z^{(q-2 m) / 2} \mathrm{~d} z \int_{0}^{\infty} e^{-z^{3} t / \omega^{2}-\omega^{2}} \frac{\mathrm{d} \omega}{\omega} .
$$


The distribution with respect to $\log a$, i.e. $F(\log a)$ is given by Equation (4) only with $x \phi(x) / 2$ replaced by $t f(t)$. The double integral in Equation (12) can be roughly evaluated with the aid of the Gaussian quadrature formula (e.g. Chandrasekhar, 1950). The distribution of $F(\log a)$ which was derived by assuming $z_{0}=0.5$ and $z_{1}=5.9$ is plotted as a solid curve in Figure 1.

While the present probing study does show that star formation in groups and the mass difference of stars both broaden the distribution function of binaries with respect to $\log a$, the general shape of the calculated curve cannot be said to agree with the observed histogram. This is especially true at large values of $a$, where the calculated curves decline sharply while the observed one shows a broad shoulder. The cause of this discrepancy is unlikely due to the neglect of variations in mass ratio $y$ and eccentricity $e$, although their inclusion in the study could somewhat modify the calculated distribution. A more important reason may well be stellar encounters which statistically widen the binary separation (Chandrasekhar, 1944; Takase, 1953). Also for very wide pairs their formation as a result of three-body encounters in interstellar space cannot be ruled out. On the other hand the slope at small values of $a$ appears to show no great divergence from that of the observed histogram. That does not mean the initial distribution of separations has not been modified by some events, such as disintegration of multiple systems, that have occurred after the formation of binaries.

From the present study and the slope of the observed histogram in Figure 1 we may draw a tentative conclusion that the origin of binaries could be dichotomic. Each binary in one group may be found from a single initial condensation in the interstellar medium. Perhaps this group centers the distribution at about $a=10 \mathrm{AU}$. The binary in the other group may be formed from two initial condensations. This means that the binary was formed by capture likely in the very early stage when stars were just emerging from the medium. The separations in the second group may have a maximum at about $a=1000 \mathrm{AU}$.

\section{Acknowledgements}

It is my pleasure to thank Mr. Clarence Wade, Jr. for his evaluation of the integrals used in the present investigation on the IBM-360 digital computer at Goddard Space Flight Center. The present investigation has been supported by a research grant from the National Aeronautics and Space Administration.

\section{References}

Abt, H.A. (1966) in Vistas in Astronomy, 8, Ed. by A. Beer and K.Aa. Strand, Pergamon Press, London, p. 75.

Chandrasekhar, S. (1944) Astrophys. J., 99, 54.

Chandrasekhar, S. (1950) Radiative Transfer, Clarendon Press, Oxford.

Chandrasekhar, S., Münch, G. (1950) Astrophys. J., 111, 142.

Chang, Y.C. (1929) Astr. J., 40, 11.

Finsen, W.S. (1933) Circ. Union Obs., 90, 397. 
Huang, S.-S. (1968) in Vistas in Astronomy (in press).

Huang, S.-S., Wade, C., Jr. (1966) Astrophys. J., 143, 146.

Hynek, J.A. (1967) in O. Struve memorial Volume (in press).

Kuch, R.H., Sobieski, S., and Wood, F.B. (1963) Pub. Univ. Pennsylvania (Astr. Series, 9).

Kuiper, G.P. (1935) Publ. astr. Soc. Pacific, 47, 15, 121.

Kuiper, G.P. (1955) Publ. astr. Soc. Pacific, 67, 387.

McCrea, W.H. (1959) Modèles d'Étoiles et Évolution Stellaire, Comm. Ninth Coll. Intern. d'Ap.,

Liège, p. 332.

Roberts, M.S. (1957) Publ. astr. Soc. Pacific, 69, 59.

Salpeter, E.E. (1955) Astrophys. J., 121, 161.

Shajn, G. (1926) Mon. Not. R. astr. Soc., 86, 543.

Shapley, H. (1948) Centennial Symposia (Harvard Obs. Monographs, 7), p. 249.

Struve, O. (1950) Sky and Telescope, 9, 216.

Takase, B. (1953) Ann. Tokyo Astr. Obs. (2), 3, 192.

\section{DISCUSSION}

J.A. Hynek: Do I understand then, that all common-proper-motion pairs are the result of later capture processes?

S.-S. Huang: According to the present study the majority of common-proper-motion pairs result from captures. However, the captures likely occurred at the time of their formation. If two stars happen to be formed near together, and if their dynamical energy turns out to be negative, the two will become a binary. Hence such a capture does not have its usual sense which requires a three-body collision. Of course for binaries with separations in the order of interstellar distances, they may be captured in the usual sense.

I.W. Roxburgh: I feel I must say a few words in defence of theoreticians. The arguments in which the angular momentum is assumed to come from galactic rotation are only done to show that there exists an angular-momentum problem. The fact that the angular-momentum vectors are distributed at random shows that the turbulence is the dominant effect and there is an even greater angular momentum problem than that obtained on using the galactic rotation.

S.-S. Huang: I agree with you that when you discuss the effect of angular momentum on stars, it does not matter where the angular momentum comes from. However, what I have emphasized in my discussion is the fact that a deep gulf of misunderstanding exists between theoretical and observational astronomers. Each group is preoccupied with its own problem without taking a look at what the other has found. Such a lack of communication perhaps is one of the serious problems in astronomy and the present one about the origin of stellar angular momentum is only one of many examples. For the sake of advancement of astronomy we should do our best to fill up such a communication gap.

L. Mestel: In discussing the formation of the initial protostars, we must certainly include not only the vorticity of the galactic rotation but that of the turbulence. In addition, the magnetic coupling between the star and a stellar wind emitted during the Hayashi phase will in general tend to rotate the angular-momentum vector.

S.-S. Huang: Magnetic coupling between the star and the stellar wind will have its effect on the stellar angular momentum just as the turbulent velocity field of the pre-stellar medium. However, one may take the coupling as another process of random nature that helps make the distribution of stellar-angular momenta Maxwellian-like.

$M$. Savedoff: Emphasize $\mathbf{H}$ field and tidal effect will reduce $\mathrm{L}$ parallel to Galaxy, especially as it was emphasized in sessions of Commission 34 that $\mathrm{H}$ field is parallel to plane. Has bias of statistics produced by a number of stars near Milky Way plane been reduced from statistics of orientation of orbital planes? This would tend to explain your figures.

S.-S. Huang: Statistical studies of orbital orientation are subject to some limitation mainly because of the difficulty of determining the sense of tilt of the orbital plane with respect to the celestial sphere. For those visual binaries whose orientation in space have been definitely determined by spectroscopic observations, their orientations appear to be at random. This is the result of Chang and of Finsen 
I quoted. Subsequently, however, other statistical studies have been performed which use many binaries whether their orientations are determined or not. Some obtained random orientation but others derived different conclusions. Those studies performed prior to 1966 have been reviewed in an article which will appear soon in Vistas in Astronomy edited by Dr. A. Beer.

A. Batten: Dommanget presented results to Commission 30 last week that suggest poles of visual binary orbits avoid the poles of the Galaxy. This must pose even worse problems than the random distribution. 